\title{
New knowledge of Philadelphia Negative Classical Myeloproliferative Neoplasms
}

\author{
Nahla Abdel Moneim Hamed* \\ Department of Hematology, Alexandria University, Egypt \\ Submission: January 31, 2017; Published: February 09, 2017 \\ "Corresponding author: Nahla Abdel Moneim Hamed, Department of Hematology, Alexandria University, Egypt
}

Abstract

This article tries to answer the following questions: why we are in need of a revision of the prior diagnostic criteria for Philadelphia -ve MPNs, what is new in 2016 multiparameter WHO classification for Philadelphia-ve MPNs and how a single JAK2V617F mutation can lead to three different MPNs. In addition it discusses the genetic basis and molecular pathophysiology of Philadelphia-ve classical myeloproliferative neoplasms.

Abbreviations: BM: Bone Marrow; CALR: Calreticulin; ET: Essential Thrombocythemia; JAK2: Janus kinase 2; MF: Myelo-Fibrosis; MPL: MyeloProliferative Leukemia virus oncogene; MPN: Myelo-Proliferative Neoplasm; PMF: Primary Myelo-Fibrosis; PV: Polycythemia Vera; WHO: World Health Organization; Hb: Hemoglobin; VWF: Von Willebrand Factor; AVWS: Acquired Von Willebrand Syndrome; HSC: Hemopoietic Stem Cell

\section{Introduction}

MPNs are a clonal disease of myeloid stem cells that are characterized by myeloid cell proliferation, BM fibrosis, and symptoms associated with the accompanying peripheral blood cell abnormalities [1]. PV is more prevalent than ET in both Europe and North America while ET was found to be the most common MPN subtype in Koreans [2].

MPNs are associated with organomegaly, cytopenia, anemia, and various constitutional symptoms [2]. Constitutional symptoms are more frequently reported in patients with MF compared to those with PV or ET. Disease-related symptoms were reported $\geq 1$ year before diagnosis in $49 \%$ of patients with MF, $61 \%$ of patients with PV and $58 \%$ of patients with ET in a landmark survey [3]. In an online survey of 1179 patients with MPN, fatigue was the most frequent symptom observed in $84 \%$ of patients with MF, $85 \%$ of patients with PV and $72 \%$ of patients with ET. Additional symptoms included were pruritis (52\%), night sweats (49\%), bone pain (44\%), fever (14\%), and weight loss (13\%). MPNs are associated with complications that can diminish quality of life such as thromboembolic events (3). Although MPNs share a relatively indolent clinical course, some patients experience progression to secondary MF, transformation to acute myeloid leukemia [2].

Why we are in need of revision of the prior diagnostic criteria for Philadelphia -ve MPNs?

i. The discovery of CALR mutation and its diagnostic importance, and influence on prognosis for all the three major entities [4]. ii. Distinguishing JAK2-mutated ET from masked PV (mPV). The term mPV was re-introduced for JAK2-mutated patients who present with disease manifestations and BM morphology consistent with PV and display persistently raised $\mathrm{Hb}$ levels between 16.0 and $18.5 \mathrm{~g} / \mathrm{dL}$ for men and 15.0 and $16.5 \mathrm{~g} / \mathrm{dL}$ for women. $\mathrm{mPV}$ is possibly under diagnosed using $\mathrm{Hb}$ levels that was established in the 2008 WHO classification for PV (between 16.0 and $18.5 \mathrm{~g} / \mathrm{dL}$ for men and 15.0 and $16.5 \mathrm{~g} / \mathrm{dL}$ for women) [5].

iii. It is necessary to differentiate "true" ET from prefibrotic/early PMF (prePMF) by lack of reticulin fibrosis in the BM biopsy at onset. Consensus among experts in the distinction between ET and pre-PMF ranges from 53\% to $88 \%$ [6].

iv. The minor clinical criteria in prePMF need to be explicitly defined since they may have a major impact on accurate diagnosis and also on prognosis.

v. Standardized morphologic criteria of MPNs are important to enhance inter observer reproducibility of morphologic diagnoses (which currently demonstrates consensus rates ranging between $76 \%$ and $88 \%$, depending on the study design) [4].

What is new in 2016 multiparameter WHO classification for Philadelphia-ve MPNs?

The 2016 multiparameter WHO classification for Philadelphia-negative MPNs integrates clinical features, 
morphology, and genetic data to diagnose PV, ET, and PMF. The main novelties are: [1] reduction of $\mathrm{Hb}$ level threshold to diagnose PV, which is now established at $16.5 \mathrm{~g} / \mathrm{dL}$ for men and $16 \mathrm{~g} / \mathrm{dL}$ for women; [2]) the recognition of prefibrotic/early PMF which is distinguishable from ET on the basis of BM morphology. Early PMF is an entity characterized by an inferior survival and a tendency to develop overt MF or acute leukemia, and; [3] the central role of BM morphology in the diagnosis of ET, prefibrotic/ early PMF, PMF, and PV with borderline Hb values; and [4] the inclusion of all mutually exclusive MPN driver mutations (JAK2, CALR, and MPL) as major diagnostic criteria in ET and PMF [6].

Genetic basis and molecular pathophysiology of classical MPNs

Mutant genes in MPNs are subdivided into MPN drivers and non-MPN drivers [7].

\section{MPN drivers (JAK2, CALR or MPL)}

a. JAK2V617F is the most frequent. It involves Janus kinase 2 (JAK2) located on chromosome 9p24. The estimated JAK2V617F mutational frequency is $97 \%$ in $\mathrm{PV}, 50-60 \%$ in ET and 55-65\% in PMF. However, JAK2V617F is not specific to MPN as it is seen in other myeloid malignancies [8]. JAK2 exon 12 mutations were described in JAK2-unmutated PV and characterized predominantly by erythroidmyelopoiesis [9]. Rare cases of JAK2-unmutated 'PV' have been associated with the inhibitory adaptor protein LNK [9].

b. The second most frequent mutations in MPN involve the CALR gene located on chromosome 19p13.2. CALR mutations frequencies are estimated at $20-25 \%$ in ET and PMF [9].

c. The third MPN-specific mutation involves the MPL; located on chromosome 1p34 and has been described in 3\% of ET and 7\% of PMF patients, and only rarely in PV [5].

d. Approximately 99\% of PV patients are expected to harbor JAK2 mutations, whereas $80-90 \%$ of patients with ET or PMF carry one of the three 'MPN-driver' mutations (JAK2, CALR or MPL). JAK2, CALR and MPL mutations are often mutually exclusive, although concurrent occurrence has been reported, likely as the result of concurrent individual mutated clones [5].

e. Cases of biclonal JAK2V617F and CALR-mutated MPNs have been reported [8].

f. About $10-15 \%$ of patients with ET or PMF lacks all three MPN driver mutations and is referred to as 'triplenegative' [5]. The search for an additional clonal marker (e.g., mutations in ASXL1, EZH2, TET2, IDH1/IDH2, SRSF2, and SF3B1) in these cases is warranted [6].

\section{Non-MPN drivers}

These include mutations in epigenetic modifiers, such as TET2, DNMT3A, ASXL1, and EZH2, and genes involved in hematopoietic signaling [10]. Mutations in the epigenetic regulators TET2 and DNMT3A are involved in disease initiation and may precede the acquisition of JAK2V617F [11]. In addition, novel recurrent mutations occurring at low frequencies in CHEK2, SCRIB, MIR662, BARD1, TCF12, FAT4, DAP3, and POLG have been also found [10].

How a single JAK2V617F mutation can lead to 3 different MPNs?

This is an intriguing question and appears to be controlled by several factors. JAK2V61F activates the three main myeloid cytokine receptors (EPOR, G-CSFR and MPL) whereas CARL or MPL mutants are restricted to MPL activation. This explains why JAK2V61F is associated with PV, ET and MF whereas CARL and MPL are found in ET and MF [11]. In JAK2/CALR/MPL-mutated patients, mutant allele burden is sometimes helpful in further clarifying the specific diagnosis; for example, higher than $40 \%$ JAK2 mutant allele burden is unusual in ET and suggests either masked PV or prefibrotic/early PMF. The disease phenotype is also influenced by the intensity of JAK2-V617F signaling (PMF greater than ET greater than PV) and by the predominant STAT activation signature [10].

\section{JAK2V617F homozygosity and a phenotypic switch in MPNs}

JAK2 mutations are not present in the normal control population. JAK2V617F homozygosity is neither sufficient for clonal expansion nor the complete phenotype observed in MPNs (e.g., leukocytosis). JAK2V617F homozygositymay provide a barrier reminiscent of oncogene-induced senescence. The underlying molecular mechanisms are unclear, but studies of single HSCs indicate that JAK2V617F impairs HSC selfrenewal while skewing their progeny toward differentiation and proliferation. Clonal expansion likely requires additional lesions. It will be critical to elucidate the mechanisms by which cooperating events drive clonal expansion of JAK2V617F mutant cells [12].

The prognostic implications of driver and non-driver mutations

JAK2/CALR/MPL mutations provide important prognostic information in MPN. A higher than 50\% JAK2V617F allele burden in PV has been associated with increased risk of fibrotic transformation. Also in PV, a higher JAK2 mutant allele burden has been associated with pruritus [5]. A low JAK2V617F allele burden has been associated with worse prognosis in PMF, and, an increased risk of thrombosis in ET (compared to those with CALR mutation or triple-negative mutational status). In ET and PMF, JAK2 mutations are associated with older age, higher $\mathrm{Hb}$ level, leukocytosis and lower platelet count [5].

The clinical course of sporadic CALR-mutated patients seems to be more indolent than that of JAK2-mutated patients [8]. In the absence of a JAK2 mutation, CALR mutations can be 


\section{Cancer Therapy \& Oncology International Journal}

associated with a PV phenotype [8]. CALR mutations in ET and PMF have been associated with younger age and higher platelet count whereas their effect on $\mathrm{Hb}$ and leukocyte count appears to be different in ET (lower $\mathrm{Hb}$ and leukocyte count) versus PMF (higher $\mathrm{Hb}$ and leukocyte levels). Furthermore, among CALRmutated ET patients, those with type 2 variant CALR mutation, display higher platelet counts compared to those with type 1 variant. More importantly, the presence of type 1 or type 1-like CALR mutations has been associated with superior survival in PMF [5].

Mutations in TP53, TET2, SH2B3 and IDH1 are more frequently observed in leukemic blasts from transformed MPN patients, suggesting a role for these gene mutations in leukemic transformation. However, so far only mutations in ASXL1 and NRAS have been shown to be of prognostic value in patients with PMF [10].

\section{What does BM examination add to our knowledge?}

BM aspirate and biopsy with trichrome and reticulin stain and BM cytogenetics (karyotype with or without FISH) is necessary to accurately distinguish the BM morphological features between the disease subtypes early/prefibrotic PMF, ET and masked PV). BM examination is often necessary to distinguish masked PV from JAK2-mutated ET, WHO defined ET from prefibrotic/early PMF or triple-negative ET from other causes of thrombocytosis. BM histology shows hypercellularity and age matched hypercellularity and megakaryocytic proliferation in most MPNs [3]. The megakaryocyte proliferation in ET is associated with large and mature morphologic features with loose cluster formation, whereas in PMF, it is associated with aberrant nuclear-cytoplasmic ratio and hyperchromatic and irregularly folded nuclei, dense clustering, granulocyte proliferation, and decreased erythropoiesis [13].

This is accompanied by a new distinction of reticulin fibrosis grade. Initial MF can only be detected by performing a BM biopsy. In PMF (grade 1 in prefibrotic/early PMF and grade 2-3 in PMF) constitute diagnostic criteria [13]. In ET, there is noor minor (grade 1) increase of reticulin fibers. However, the presence of reticulin fibrosis grade 1 is a very rare presentation. Some PV patients (20\%) may have grade 1 reticulin fibrosis in the BM at diagnosis. This does not per se imply a diagnosis of MF but notably is associated with a higher risk of secondary MF [6]. BM biopsy may not be required in patients with absolute erythrocytosis and $\mathrm{Hb}$ levels $\geq 18.5 \mathrm{~g} / \mathrm{dl}$ in males (Ht: $55.5 \%$ ) or $\mathrm{Hb}>16.5 \mathrm{~g} / \mathrm{dl}$ in women (Ht: 49.5), JAK2 V617F or JAK2 exon 12 mutations and subnormal EPO level [3]. However, marrow biopsy becomes mandatory for the diagnosis of PV with a normal EPO level [14].

\section{Treatment}

Treatment is based on the risk stratification. Riskstratification models have always been remarkably simple for ET and PV, two of the lower-risk subtypes of MPNs - perhaps due to the limited number of therapeutic interventions employed.
However, in PMF, a disease where survival can range from months to over a decade, there has been continuous re-evaluation of the prognostic models used [15].

One notable and unique recommendation in patients with MPNs is to perform a functional characterization of VWF, including Ristocetin Cofactor activity (VWF: RCo), VWF activity and VWF collagen binding activity (VWF: CB), etc., in patients with more than $1000 \mathrm{X} 10^{9} / \mathrm{L}$ platelets in order to assess the extent of secondary VWF deficiency. Interestingly, AVWS was found to occur at near-normal platelet counts in a patient with PV. ASA should be avoided if platelet count is $>1000 \mathrm{X} 10^{\circ} / \mathrm{L}$ or if the VWF: RCo is $\leq 30 \%$. Of note, the ability of VWF testing to reliably predict the risk of bleeding is limited [16].

None of the available drugs has shown clear evidence of disease modifying activity. Interferon alfa was able to reduce the JAK2-mutated clone and to induce molecular complete responses in some patients with MPNs [17]. Responses to IFN have also been recently reported in CALR-mutated ET patients. Analyses of mutation type suggested a differential effect from IFN-alpha on mutated clones, because some patients experienced a decrease in the CALR burden but an increase in the clonal burden of other somatic mutations [18].

There has been a major revolution in the management of patients with MPN in particular those with MF and extensive splenomegaly and symptomatic burden following the introduction of JAK1 and JAK2 inhibitor ruxolitinib. The drug has been later approved as a second line therapy for PV. However the therapeutic armamentarium for MPN is still largely inadequate to prevent hematological progression and normalize patients' life span [19].

\section{References}

1. Kim SY, Im K, Park SN, Kwon J, Kim JA, et al. (2015) CALR, JAK2, and MPL mutation profiles in patients with four different subtypes of myeloproliferative neoplasms: primary myelofibrosis, essential thrombocythemia, polycythemia vera and myeloproliferative neoplasm, unclassifiable. Am J Clin Pathol 143(5): 635-644.

2. Byun JM, Kim YJ, Youk T, Yang JJ, Yoo J, et al. (2016) Real world epidemiology of myeloproliferative neoplasms: a population based study in Korea 2004-2013. Ann Hematol 96(3): 373-381.

3. NCCN Clinical Practice Guidelines in Oncology 2017 (NCCN guidelines): Myeloproliferative Neoplasms. Version 2.2017.

4. Daniel A Arber, Attilio Orazi, Robert Hasserjian, Jürgen Thiele, Michael J Borowitz, et al. (2016) The 2016 revision to the World Health Organization classification of myeloid neoplasms and acute leukemia. Blood 127(20): 2391-2405.

5. Barbui T, Thiele J, Vannucchi AM, Tefferi A (2015) Rationale for revision and proposed changes of the WHO diagnostic criteria for polycythemia vera, essential thrombocythemia and primary myelofibrosis. Blood Cancer J 5(8): e337.

6. Passamonti F, Maffioli M (2016) Update from the latest WHO classification of MPNs: a user's manual. Hematology Am Soc Hematol Educ Program 2016(1): 534-542.

7. Cazzola M (2016) Introduction to a review series on myeloproliferative neoplasms. Blood. 


\section{Cancer Therapy \& Oncology International Journal}

8. Broseus J, Park JH, Carillo S, Hermouet S, Girodon F (2014) Presence of calreticulin mutations in JAK2-negative polycythemia vera. Blood 124(26): 3964-3966.

9. Oh ST, Simonds EF, Jones C, Hale MB, Goltsev Y, et al. (2010) Novel mutations in the inhibitory adaptor protein LNK drive JAK-STAT signaling in patients with myeloproliferative neoplasms. Blood 116(6): 988-992.

10. Silvennoinen O, Hubbard SR (2015) Molecular insights into regulation of JAK2 in myeloproliferative neoplasms. Blood 125(22): 3388-3392.

11. Vainchenker W, Kralovics R (2016) Genetic basis and molecular pathophysiology of classical myeloproliferative neoplasms. Blood.

12. Li J, Kent DG, Godfrey AL, Manning H, Nangalia J, et al. (2014) JAK2V617F homozygosity drives a phenotypic switch in myeloproliferative neoplasms, but is insufficient to sustain disease. Blood 123(20): 31393151.

13. Tefferi A, Barbui $T$ (2015) Essential thrombocythemia and polycythemia vera: Focus on clinical practice. Mayo Clin Proc 90(9): 1283-1293.

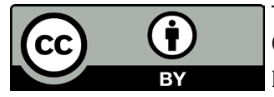

This work is licensed under Creative Commons Attribution 4.0 License

DOI: 10.19080/CTOIJ.2017.03.555604
14. Silver RT, Chow W, Orazi A, Arles SP, Goldsmith SJ (2013) Evaluation of WHO criteria for diagnosis of polycythemia vera. A prospective analysis. Blood 122(11): 1881-1886.

15. Laura C Michaelis (2017) Risk stratification in myelofibrosis: the quest for simplification. Haematol 102(1): 2-3.

16. Falchi L, Bose P, Newberry KJ, Verstovsek S (2016) Approach to patients with essential thrombocythemia and very high platelet counts: what is the evidence for treatment? Br J Haematol 176(3): 352-364.

17. Cassinat B, Verger E, Kiladji JJ (2014) Interferon alfa therapy in CALRMutated essential thrombocythemia. N Engl J Med 371(2): 188-189.

18. Shammo JM, Stein BL (2016) Mutations in MPNs: prognostic implications, window to biology, and impact on treatment decisions. Hematology Am Soc Hematol Educ Program 2016(1): 552-560.

19. Vannucchi AM, Harrison C (2016) Emerging treatments for classical myeloproliferative neoplasms. Blood.

\section{Your next submission with Juniper Publishers} will reach you the below assets

- Quality Editorial service

- Swift Peer Review

- Reprints availability

- E-prints Service

- Manuscript Podcast for convenient understanding

- Global attainment for your research

- Manuscript accessibility in different formats

( Pdf, E-pub, Full Text, Audio)

- Unceasing customer service

Track the below URL for one-step submission https://juniperpublishers.com/online-submission.php 